\title{
Clinical utility gene card for: Haemophilia A
}

\author{
Steve Keeney ${ }^{\star,}$, Tony Cumming ${ }^{1}, \mathrm{P}$ Vincent Jenkins ${ }^{2}$, James S O’Donnell ${ }^{2}$ and Michael J Nash ${ }^{1}$ \\ European Journal of Human Genetics (2011) 19, doi:10.1038/ejhg.2011.107; published online 8 June 2011
}

\section{DISEASE CHARACTERISTICS}

1.1 Name of the disease (synonyms)

Haemophilia A (F8 deficiency).

1.2 OMIM\# of the disease

306700.

1.3 Name of the analysed genes or DNA/chromosome segments F8 (Xq28).

\subsection{OMIM\# of the gene(s)}

306700 .

\subsection{Mutational spectrum}

Approximately $45 \%$ of severe Haemophilia A is accounted for by a large gene inversion mediated via intron 22 of F8. A further $2-3 \%$ of severe disease is mediated via an inversion mechanism involving intron 1 . The remaining severe and mild/moderate disease is due mainly to point mutations throughout the 26 exons of the gene. Indels (large or small) account for a minority of Haemophilia A cases. A locus-specific database for Haemophilia A, including information on reported mutations, can be found at http://hadb.org.uk/.

\subsection{Analytical methods}

Long PCR or 'inverse' PCR for detection of intron 22 mediated inversion. $^{1,2}$ Standard PCR or 'inverse' PCR for detection of the intron 1 mediated inversion. ${ }^{2-4}$

DNA sequencing of the essential regions of F8. Multiplex ligationdependent probe amplification (MLPA) can be applied to detection of rarer deletions or insertions. ${ }^{5,6}$

\subsection{Analytical validation}

Recommended practice including analytical design, mutation validation procedures, and analytical pitfalls is discussed in a current practice guideline for the molecular analysis of Haemophilia A. ${ }^{7}$ External quality assurance (EQA) should be carried out where available. An EQA scheme has been established for genetic investigation of Haemophilia (and von Willebrand disease (VWD)), details of this scheme are available from UK NEQAS for blood coagulation. ${ }^{8} \mathrm{~A}$ World Health Organisation international standard gDNA reference material panel for the F8 intron 22 inversion mutation is available from the UK National Institute for Biological Standards and Control (NIBSC). ${ }^{9}$ Internal quality control can be performed using anonymous reference mutants.
1.8 Estimated frequency of the disease (incidence at birth ('birth prevalence') or population prevalence) 1 in 5000 males.

1.9 If applicable, prevalence in the ethnic group of investigated person

Not applicable.

\subsection{Diagnostic setting}

\begin{tabular}{lcc}
\hline & Yes & No \\
A. (Differential) diagnostics & $\bigotimes$ & $\square$ \\
B. Predictive testing & $\square$ & $\square$ \\
C. Risk assessment in relatives & $\bigotimes$ & $\square$ \\
D. Prenatal & $\square$ & $\square$ \\
\hline
\end{tabular}

Comment: Haemophilia $\mathrm{A}$ is an X-linked recessive bleeding disorder. Establishing if a bleeding disorder is associated with F8 is normally achieved by measurement of functional coagulation factor VIII levels (FVIII:C) using standard coagulation tests. The carrier status of females can be determined once the familial mutation has been identified in an affected male index case.

Differential diagnosis applies to confirmation of a moderate/mild Haemophilia A phenotype being linked to F8. Type 2N VWD is a rare condition in which the phenotype is near identical to mild or moderate Haemophilia A. Type $2 \mathrm{~N}$ VWD is an autosomal recessive disorder which can be diagnosed or excluded by FVIII/VWF binding studies or analysis of the VWF locus. ${ }^{10}$ Acquired Haemophilia A, a late onset condition, is not linked to F8.

Predictive testing does not apply as males with a F8 mutation will be affected.

Risk assessment in relatives relates to genetic testing of females at risk of carriership. In general, carrier diagnosis is performed when requested after identification of the familial mutation in the index case. Prenatal diagnosis (PND) is available when requested; pre-implantation genetic diagnosis (PGD) has been performed for Haemophilia A. Knowledge of carrier status can inform clinical management of childbirth. 


\section{TEST CHARACTERISTICS}

\begin{tabular}{|c|c|c|c|c|}
\hline & \multicolumn{2}{|c|}{ Genotype or disease } & \multirow{2}{*}{$\begin{array}{l}\text { A: True positives } \\
\text { B: False positives }\end{array}$} & \multirow{2}{*}{$\begin{array}{l}\text { C: False negatives } \\
\text { D: True negatives }\end{array}$} \\
\hline & Present & Absent & & \\
\hline \multicolumn{5}{|l|}{ Test } \\
\hline \multirow[t]{2}{*}{ Positive } & $A$ & $\mathrm{~B}$ & Sensitivity: & $A /(A+C)$ \\
\hline & & & Specificity: & $D /(D+B)$ \\
\hline \multirow[t]{2}{*}{ Negative } & $\mathrm{C}$ & $\mathrm{D}$ & Positive predictive value: & $A /(A+B)$ \\
\hline & & & Negative predictive value: & $\mathrm{D} /(\mathrm{C}+\mathrm{D})$ \\
\hline
\end{tabular}

2.1 Analytical sensitivity (proportion of positive tests if the genotype is present) Greater than $99 \%$.

\subsection{Analytical specificity}

(proportion of negative tests if the genotype is not present)

Greater than $99 \%$.

\subsection{Clinical sensitivity}

(proportion of positive tests if the disease is present)

The clinical sensitivity can be dependent on variable factors such as age or family history. In such cases, a general statement should be given, even if a quantification can only be made case by case.

An estimated $98 \%$ for severe cases, however, mutations have not been identified in an estimated $2-18 \%$ of Haemophilia A patients overall, after exclusion of inversion events and mutations in the essential regions of F8. ${ }^{11-17}$ This proportion may vary according to the accuracy of the initial Haemophilia A diagnosis, particularly in milder cases, and the sensitivity of the mutation detection protocol in use. Modifying factors may affect the clinical severity of the phenotype to some extent, eg, co-inheritance of FV Leiden.

\subsection{Clinical specificity}

\section{(proportion of negative tests if the disease is not present)}

The clinical specificity can be dependent on variable factors such as age or family history. In such cases, a general statement should be given, even if a quantification can only be made case by case.

Genetic screening is generally only performed on males with a diagnosis of Haemophilia A based on their FVIII:C level. Heterozygous female carriers do not exhibit the disease although $\sim 10 \%$ of 'low level' carriers may exhibit mild bleeding symptoms. ${ }^{18}$ Occasionally genetic screening for identification of the familial mutation is performed on an obligate carrier female where a sample from an affected male in the kindred is not available.

\subsection{Positive clinical predictive value}

(life-time risk of developing the disease if the test is positive)

Not applicable as condition present from birth and the primary diagnosis is made on phenotypic analysis.

\subsection{Negative clinical predictive value}

(probability of not developing the disease if the test is negative)

Assume an increased risk based on family history for a non-affected person. Allelic and locus heterogeneity may need to be considered.

Index case in that family had been tested:

Not applicable - see 2.5 above.

Index case in that family had not been tested: Not applicable - see 2.5 above.

\section{CLINICAL UTILITY}

3.1 (Differential) diagnosis: The tested person is clinically affected (To be answered if in 1.10 'A' was marked)

\subsubsection{Can a diagnosis be made other than through a genetic test?}

\begin{tabular}{|c|c|c|}
\hline No & $\square$ (continue with 3.1.4) & \\
\hline Yes & $\nabla$ & \\
\hline & Clinically & $\nabla$ \\
\hline & Imaging & $\square$ \\
\hline & Endoscopy & $\square$ \\
\hline & Biochemistry & $\otimes$ \\
\hline & Electrophysiology & $\square$ \\
\hline & Other (please describe) & $\begin{array}{l}\otimes \text { Standard coagulation testing of FVIII:C level in } \\
\text { affected males. Carrier females cannot be reliably } \\
\text { diagnosed by this means }\end{array}$ \\
\hline
\end{tabular}

3.1.2 Describe the burden of alternative diagnostic methods to the patient

Low for index males (blood collection). Carrier females cannot be definitively diagnosed by phenotypic means.

3.1.3 How is the cost effectiveness of alternative diagnostic methods to be judged?

Primary diagnosis in affected males achieved by measurement of FVIII:C levels. Identifying the mutation in an index male allows carrier detection in females (who cannot be diagnosed by phenotypic means) and, in severe Haemophilia A cases, subsequent PND or appropriate management of the birth. Genetic diagnosis is stratified depending on the severity of the disorder (see section 1.5). Approximately $48 \%$ of severe cases are accounted for by either the intron $22(\sim 45 \%)$ or intron 1 inversion $(\sim 3 \%)$ mutations which can be screened for directly. The remaining severe cases and all mild and moderate cases require DNA sequence analysis of the essential regions of F8 in order to identify the underlying mutation.

3.1.4 Will disease management be influenced by the result of a genetic test?

No $\square$

Yes $\otimes$

Therapy Replacement FVIII concentrate therapy is the standard treatment (please and in general is not influenced by mutation. However, in certain describe) instances modulation of treatment dosage and type may be influenced by knowledge of the underlying mutation (see management section below).

Prognosis Prognosis is unaffected.

(please

describe)

Management Certain mutations are associated with an increased risk of (please antibody formation (inhibitors) against FVIII replacement condescribe) centrates. Overall, large deletions show the highest risk, while missense mutations and splicing defects show the lowest risk However, an important consideration when reporting mutations associated with inhibitor formation is whether or not knowledge of this increased risk may influence treatment strategy when using replacement therapies. ${ }^{7}$ Treatment strategy is unlikely to be affected where the mutation results in severe Haemophilia A. A small number of missense mutations associated with mild or moderate Haemophilia A and a particularly increased risk of inhibitor formation have been identified. ${ }^{7}$ Knowledge of the presence of these mutations may influence treatment in mild or moderate Haemophilia in order to minimise the risk of inhibitor formation. 
3.2 Predictive setting: The tested person is clinically unaffected but carries an increased risk based on family history

(To be answered if in 1.10 ' $\mathrm{B}$ ' was marked)

\subsubsection{Will the result of a genetic test influence lifestyle and prevention?}

If the test result is positive (please describe):

No influence for affected males (diagnosis made phenotypically based on FVIII:C levels).

If a female is confirmed to be a carrier of severe Haemophilia A this may influence family planning decisions, eg, PND. If not already ascertained, a positive genetic test may lead on to establish if a female is a low level carrier by phenotypic means.

If the test result is negative (please describe):

A negative result in a female from a Haemophilia A family provides reassurance with regard to non-carrier status and removes concerns related to family planning.

3.2.2 Which options in view of lifestyle and prevention does a person at-risk have if no genetic test has been done (please describe)?

Males will be managed on their clinical presentation. Female query carriers with a low FVIII level may require management during at-risk procedures.

\subsection{Genetic risk assessment in family members of a diseased} person

(To be answered if in 1.10 ' $\mathrm{C}$ ' was marked)

\subsubsection{Does the result of a genetic test resolve the genetic situation in that family?}

Yes.

\subsubsection{Can a genetic test in the index patient save genetic or other} tests in family members?

Identification of the mutation in an index case characterises the mutation in that family and may avoid testing of other affected males. It does not preclude screening for the familial mutation in possible carrier females wishing to definitively know their carrier status.

\subsubsection{Does a positive genetic test result in the index patient enable a} predictive test in a family member?

In general, affected males are identified by clinical presentation and FVIII:C levels.

\subsection{Prenatal diagnosis}

(To be answered if in 1.10 ' $\mathrm{D}$ ' was marked)

\subsubsection{Does a positive genetic test result in the index patient enable a prenatal diagnosis?}

Yes. PND, if required, would only be performed on a male fetus. Normally this would only be considered in families with severe Haemophilia A, or in some cases of moderate Haemophilia A where there is a severe bleeding tendency. In developed countries, advances in patient management and the nascent use of PGD have resulted in PND for Haemophilia A being requested less frequently. Developing countries, which do not have ready access to FVIII replacement therapy, still have a high request rate for PND.

\section{IF APPLICABLE, FURTHER CONSEQUENCES OF TESTING}

Please assume that the result of a genetic test has no immediate medical consequences. Is there any evidence that a genetic test is nevertheless useful for the patient or his/her relatives? (Please describe)

Analysis of female carrier status is often requested due to the psychosocial implications of Haemophilia on a carrier and family. Knowing the carrier status in females who are pregnant with a male fetus may influence the degree of planned medical intervention during delivery. Genetic testing may be useful for prediction of inhibitor formation risk in mild or moderately affected males (see section 3.14).

\section{CONFLICT OF INTEREST}

The authors declare no conflict of interest.

\section{ACKNOWLEDGEMENTS}

This work was supported by EuroGentest, an EU-FP6 supported NoE, contract number 512148 (EuroGentest Unit 3: 'Clinical genetics, community genetics and public health', Workpackage 3.2 ).

1 Liu Q, Sommer SS: Subcycling-PCR for multiplex long distance amplification of regions with high and low GC content: application to the inversion hotspot in the FVIII gene. Biotechniques 1998; 25: 1022-1028.

2 Rossetti LC, Radic CP, Larripa IB, De Brasi CD: Developing a new generation of tests for genotyping hemophilia causative rearrangements involving int22 $\mathrm{h}$ and int $1 \mathrm{~h}$ hotspots in the factor 8 gene. J Thromb Haemost 2008; 6: 830-836.

3 Bagnall RD, Waseem N, Green PM, Giannelli F: Recurrent inversion breaking intron 1 of the factor VIII gene is a frequent cause of severe hemophilia A. Blood 2002; 99: 168-174.

4 Rossetti LC, Radic CP, Larripa IB, De Brasi CD: Genotyping the hemophilia inversion hotspot by use of inverse PCR. Clin Chem 2005; 51: 1154-1158.

5 Lannoy N, Abinet I, Dahan K, Hermans C: Identification of de novo deletion in the factor VIII gene by MLPA technique in two girls with isolated factor VIII deficiency. Haemophilia 2009; 15: 797-801.

6 Santacroce R, Longo V, Bafunno V et al: Detection of new deletions in a group of Italian patients with Hemophilia A by multiplex ligation-dependent probe amplification. Genet Test Mol Biomarkers 2009; 13: 573-576.

7 Keeney S, Mitchell M, Goodeve A: UKHCDO/CMGS Best Practice Guidelines for the molecular analysis of Haemophilia A: http://www.cmgs.org/BPGs/Best_Practice_Guidelines.htm.

8 EQA scheme for genetic investigation of haemophilia and VWD; UK NEQAS for Blood Coagulation. http://www.ukneqasbc.org/content/PageServer.asp?S=932234149\&C= $1252 \& \mid D=32$.

9 NIBSC. The National Institute for Biological Standards and Control, produces the reference material panel for the F8 intron 22 inversion mutation. http://www.nibsc.ac.uk/.

10 Keeney S, Bowen D, Cumming A, Enayat S, Goodeve A, Hill M: UKHCDO/CMGS Best Practice Guidelines for the molecular analysis of VWD. http://www.cmgs.org/BPGs/ Best Practice Guidelines.htm.

11 Oldenburg J, Ivaskevicius V, Rost S et al: Evaluation of DHPLC in the analysis of hemophilia A. J Biochem Biophys Methods 2001; 47: 39-51.

12 Klopp N, Oldenburg J, Uen C, Schneppenheim R, Graw J: 11 hemophilia A patients without mutations in the factor VIII encoding gene. Thromb Haemost 2002; 88 : 357-360.

13 Jayandharan G, Shaji RV, Baidya S, Nair SC, Chandy M, Srivastava A: Identification of factor VIII gene mutations in 101 patients with haemophilia A: mutation analysis by inversion screening and multiplex PCR and CSGE and molecular modelling of 10 novel missense substitutions. Haemophilia 2005; 11: 481-491.

14 Vinciguerra C, Zawadzki C, Dargaud Y et al: Characterisation of 96 mutations in 128 unrelated severe haemophilia A patients from France. Description of 62 novel mutations. Thromb Haemost 2006; 95: 593-599.

15 Bogdanova N, Markoff A, Pollmann $\mathrm{H}$ et al: Spectrum of molecular defects and mutation detection rate in patients with severe hemophilia A. Hum Mutat 2005; 26 : 249-254

16 Bogdanova N, Markoff A, Eisert R et al: Spectrum of molecular defects and mutation detection rate in patients with mild and moderate hemophilia A. Hum Mutat 2007; 28: 54-60.

17 Santacroce R, Acquila M, Belvini D et al: Identification of 217 unreported mutations in the F8 gene in a group of 1,410 unselected Italian patients with hemophilia A. J Hum Genet 2008; 53: 275-284.

18 Plug I, Mauser-Bunschoten EP, Bröcker-Vriends AH, van Amstel HK, van der Bom JG, van Diemen-Homan JE: Bleeding in carriers of haemophilia. Blood 2006; 108: 52-56. 